\title{
Complex Detection and Subtraction via Wavelet, a New Atrial Activity Extracting Algorithm
}

\author{
C Aguilar ${ }^{1}$, C Sanchez ${ }^{1}$, JJ Rieta $^{2}$, D Moratal-Perez ${ }^{2}$, \\ C Vaya ${ }^{2}, \mathrm{JM}$ Blas $^{1}, \mathrm{~J}_{\text {Millet }}{ }^{2}$ \\ ${ }^{1}$ Innovation in Bioengineering, University of Castilla-La Mancha, Cuenca, Spain \\ ${ }^{2}$ Bioengineering, Electronics and Telemedicine, Valencia University of Technology, Spain
}

\begin{abstract}
In this paper, a new technique for extracting the Atrial Activity (AA) using a single-lead from surface ECG and based on Wavelet transform and adaptive filtering, is presented. Firstly, the fiducial points of each beat are detected using a Discrete Wavelet Transform (DWT). In the second stage, the dominant frequency ( $F p)$ of the $f$ waves segments is calculated, allowing the application of an adaptive filtering. Averaging this signal with a median complex based on Template Matching and Subtraction cancellation technique (TMS) results a signal where $A A$ is minimum. Finally, a subtraction between the original lead and the averaged signal produces a residual signal which contains the expected AA. The presented results show that Complex Detection and Subtraction via Wavelet (CDSW) can be a highly efficient tool for the study of atrial arrhythmias in those systems with reduced number of leads, like Holter recording systems.
\end{abstract}

\section{Introduction}

Atrial Fibrillation (AF) is one of the most important cardiac diseases, affecting to a vast sector of the population, ancient specially. Approximately, the $34.5 \%$ of patients hospitalized with cardiac rhythm alterations are caused by AF. The mechanisms behind AF have not been completely understood [1].

Historically, the study of AF has been based on invasive methods, which implies costs and risks. Recent studies are focused on non invasive methods, mainly from the standard 12 lead ECG, a simply, low cost and clean diagnostic tool.

The drawback of using the ECG to analyse such fibrillation is the spectral and temporal concealment of the Atrial Activity (AA) by the Ventricular Activity (VA). This fact makes necessary the development of new non invasive techniques for extracting the AA from ECG recordings.

Actually, there are different separation and extraction techniques which can be classified according to the number of required leads [2]. This work has been focused on achieving a single-lead based solution to reduce the problem size and the processing time.

Complex Detection and Subtraction via Wavelet (CDSW) is based on the interpretation of the ECG lead as is proposed in [3], this is, noise, AA and VA. The residual signal, identified as AA, is obtained by minimizing noise from original lead and shaping a VA signal which is subtracted to the original.

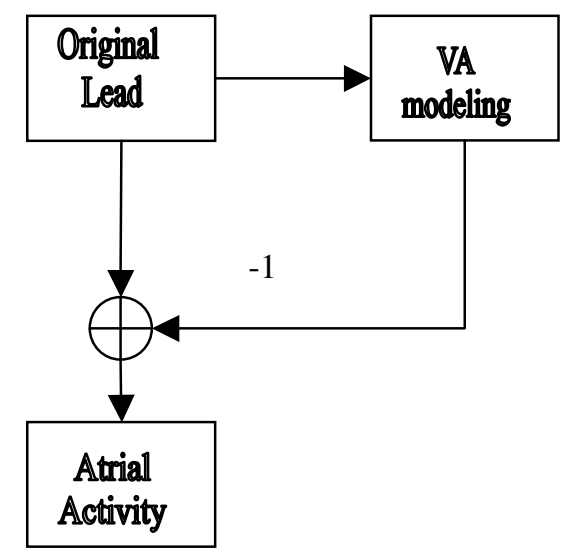

Figure 1. Process of the CDSW method.

\section{Methods}

CDSW has been developed using a large database. This is composed of about 80 registers, including AF real episodes and artificial signals.

The real ECG recordings have been obtained from the Electrophysiology's Laboratory database of the Valencia's University Hospital. The database consists of 48 standard 12 lead registers in a time of 12 seconds and a sampling frequency of $1 \mathrm{KHz}$. All of them have been catalogued like AF episodes by experimented cardiologist.

ECG recordings from the series 100 and 200 of the MIT's Arrhythmia Database have been used.

Artificial signals, where AA was known, have been generated, and the accuracy of the extraction method can 
be calculated.

The registers have been synthesized by means of the algorithm of McSharry \& Clifford [4]. Then, a signal generated by the proposed equation in [3] was added to the previous signal.

Table 1. Procedence and number of ECG registers.

\begin{tabular}{cc}
\hline Procedente & Number of Registers \\
\hline Valencia's University & 48 \\
Hospital & 20 \\
MIT. Arrhythmia Database & 12 \\
Synthetized Signals & \\
\hline
\end{tabular}

The higher presence of AF in the limb lead II [1], has led to use this signal from surface ECG recordings.

CDSW process can be divided in four main parts: preprocessing, detection of the characteristic wave points, spectral analysis and obtaining the AA signal by means of a subtraction process.

\subsection{Pre-Processing}

A straightforward band-pass filter was applied from 0.5 to $40 \mathrm{~Hz}$ to remove powerline interference, high frequency and thermal noise and eventual artifacts. The baseline wandering was removed using an alignment algorithm [5].

\subsection{Characteristic Points Detection}

The Wavelet Transform allows to obtain time-scale representations, being a very useful tool for providing information about frequency variations in ECG recordings with precise location in time. The detection of the QRS complex was carried out by means of a Discrete Wavelet transform. After testing different functions, the best results were obtained using a biorthogonal Mother Wavelet at scale $2^{1}$.

After that, the normalized and squared signal from the detail coefficients is used in a thresholding process based on Stein's Unbiased Risk Estimation Principle (SURE), removing the non-relevant information like noise and artifacts.

The fiducial point is identified as the local maxima, and the zero crosses in the adjacent lateral bands are identified as the $\mathrm{Q}$ and $\mathrm{S}$ waves.

\subsection{Spectral Analysis}

In order to design a customized filtering system, the Power Spectral Density (PSD) is measured for each fwaves segment. The analysis is carried out by means of the Welch-WOSA method with a Hamming window length of 4096 points, an overlapping of $50 \%$ between windows and a FFT of 8192 points of length [5].
Once the PSD is calculated, the maximum value is used to identify the dominant frequency of each section.

This methodology of frequency analysis is also applied to the AA extracted signal for its characterization, verifying the morphology of the PSD and the dominant frequencies.

(a)

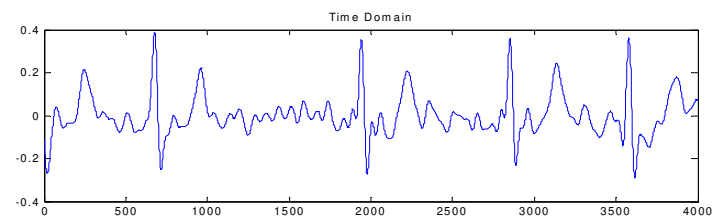

(b)

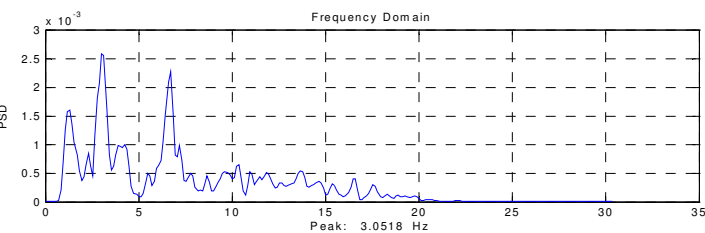

Figure 2. (a) Original Lead DII. (b) PSD of the original signal.

\subsection{Obtaining the signal}

Initially, the extraction of the AA was performed using a normalized LMS filter. The input reference signal was generated from the f-waves segments but several inconvenients were found. The reference signal was a poor estimation of the real AA, the adaptation of the filter coefficients wasn't successful, producing unexpected distortions and artifacts in the extracted signal.

Finally, this stage was carried out by means of filtering, averaging and subtracting methods. The modeling of the VA signal is achieved in two stages. In the first stage, every QQ interval is filtered, removing the frequencies on the band $[\mathrm{Fp}-0.6, \mathrm{Fp}+0.6] \mathrm{Hz}$, where $\mathrm{Fp}$ is the dominant frequency of the associated f-waves segment.

In the second stage, a median complex based on Template Matching and Subtraction technique (TMS) is constructed [6]. TMS is the most referenced classical technique of subtraction. It's based on aligning all the detected QRS complexes in the register at their fiducial point and averaging them. This operation produces the median complex which is used for a subtraction, producing the remainder signal which is identified as AA.

The averaging of both signals yields a modeled VA signal which avoids the $\mathrm{T}$ wave truncations, eliminates the correlated AA and restores the frequencies of the AV that were eliminated in the filtering.

Subsequently, the subtraction between the modeled VA signal and the original leads produces a residual signal that is identified as the expected AA.

The maximum information of AA is concentrated on the band from 4 to $9 \mathrm{~Hz}$ [6], being found the frequency 
peak between 5 and $7 \mathrm{~Hz}$, which can be observed in the figure 3:

(a)

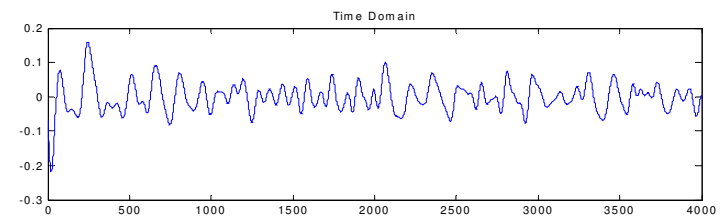

(b)

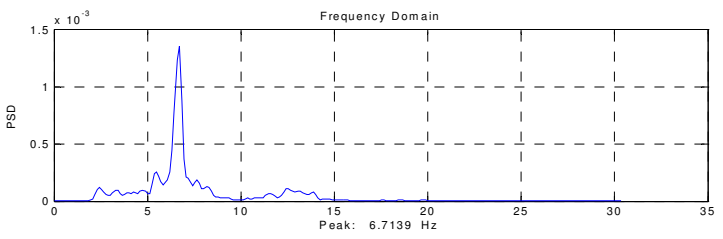

Figure 3. (a) Extracted AA (b) PSD of the extracted AA.

\section{Results}

In the development of the method, the fact of achieving an extracted signal without discontinuities, $\mathrm{T}$ wave presence, truncated complexes and others artefacts that inhibit the correct AA study has been emphasized.

In order to qualify the validity of the method, some relevant parameters have been selected [7]. In temporal domain, the correlation parameter (Corr-T) was measured. In spectral domain the selected parameters are the amplitude (Ap) and frequency (Fp) of the main peak, the First Spectral Moment Normalized (FSMN), distribution and relation of energy in different bands (A1, PPBP), the first and second order moments (M1, M2) and the correlation coefficients (Corr-F).

It should be indicated that the measurement of Corr-T and Corr-F was applied to the synthetic signals with known AA. In the case of the real signals, the coefficients of temporal and spectral correlation were calculated in the TQ intervals and were compared with the extracted signal, obtaining a correlation upper to $80 \%$ in the time domain and upper to $90 \%$ in frequency domain.

The obtained results are shown in the Table 2:

Table 2. Spectral and temporal results.

\begin{tabular}{cccc}
\hline Parameters & Original AA & CDSW & TMS \\
\hline Fp & 7.8125 & 7.8125 & 7.9346 \\
Ap & 4.6334 & 3.8026 & 4.0434 \\
FSMN & 0.7289 & 0.5992 & 0.5177 \\
A1 & 0.7739 & 0.7601 & 0.8350 \\
PPBP & 0.1690 & 0.1622 & 0.1124 \\
M1 & 0.7324 & 0.7324 & 0.8545 \\
M2 & 0.0099 & 0.0099 & 0.0099 \\
Corr-F & $100 \%$ & $91.17 \%$ & $84.77 \%$ \\
Corr-T & $100 \%$ & $84.05 \%$ & $79.44 \%$ \\
\hline
\end{tabular}

For evaluating the suggested algorithm, the results have been compared with the TMS technique. This comparison reports that the new cancellation method performs better than does the classical technique, avoiding the presence of ectopics complexes and residual waves from the cancellation (e.g. truncated complexes or artifacts [6]).

\section{Discussion and conclusions}

The presented results show that CDSW can be a highly efficient tool for the study of atrial arrhythmias in systems of collecting with a number reduced of derivations, like Holter systems.

CDSW presents a better adaptation to the QRS complex morphology and its variations (such as premature complexes).

Further improvements of CDSW are focused on providing more information for methods that requires more reference sources.

\section{Acknowledgements}

This work was partly funded by several research grants from Junta de Comunidades de Castilla-La Mancha (Ref: PAC-05-008-1) and Consellería de Empresa Universidad y Ciencia de la Generalitat Valenciana (Ref: IIARC0/2004/249).

\section{References}

[1] Fuster V, et al. ACC/AHA/ESC Guidelines for the management of patients with atrial fibrillation: executive summary. Journal of the American College of Cardiology 2001;38(4):1266i-1266lxx.

[2] Langley P, Rieta JJ, Stridh M, Millet J, Sörnmo L, Murray A. Reconstruction of Atrial Signals Derived from 12-lead ECG using Atrial Signal Extraction Techniques. IEEE Computers in Cardiology 2003; 30:129-132.

[3] Sörnmo L. Spatiotemporal QRST Cancellation Techniques for Analysis of Atrial Fibrillation. IEEE Trans. Biomed. Eng 2001;48(1):105:111.

[4] McSharry PE, Clifford GD, Tarassenko L, Smith LA. A Dynamical Model for Generating Synthetic Electrocardiogram Signals. IEEE Trans BioMed Eng 2003;289:294-50.

[5] Rieta JJ, Castells F, Sanchez C, Zarzoso V, Millet J. Atrial Activity Extraction for Atrial Fibrillation Analysis Using Blind Source Separation. IEEE Trans Biomed Eng 2004;51(7):1176-1186.

[6] Shkurovich S, Sahakian AV, Swiryn S. Detection of Atrial Activity from High-Voltage Leads of Implantable Ventricular Defibrillators Using a Cancellation Technique. IEEE Trans Biomed Eng 1998;45(2):229-234.

[7] Barro S, Ruiz R, Cabello D, Mira J. Algorithmic sequential decision-making in the frequency domain for life threatening ventricular arrhythmias and imitiative artifacts: a diagnostic system. Journal Biomedical Engineering 1989; 11: $320-328$. 
[8] Martínez JP, Almeida R, Olmos S, Rocha AP, Laguna P. A Wavelet-Based ECG Delineator: Evaluation on Standard Databases. IEEE Trans BioMed Eng 2004; 570:581-51.

[9] Stridh M, Sörnmo L, Meurling CJ and Olsson SB. Characterization of Atrial Fibrillation Using the Surface ECG: Time-Dependent Spectral Properties. IEEE Trans Biomed Eng 2001;48(1):19-27.
Address for correspondence:

Carlos Aguilar Palacios

EUP Cuenca. Universidad de Castilla-La Mancha. Campus Universitario s/n. 16071. Cuenca (Spain) datos_proyecto@yahoo.es 